\title{
A Portable Remote Medical Consultation System for the Use of Distant Rural Communities
}

\author{
Sisil P. Kumarawadu ${ }^{1}$, Nuwan D Nanayakkara ${ }^{1}$, Brinthan Thavaneswaran ${ }^{1}$, Mukunthan
}

Tharmakulsingam ${ }^{* 1}$, Nirosan Paramanathan ${ }^{1}$, Lalitha Perera ${ }^{1}$ and Sajith Mohamed ${ }^{2}$

\author{
${ }^{1}$ University of Moratuwa, Sri Lanka. \\ ${ }^{2}$ Arthur C Clarke Institute for Modern Technologies, Sri Lanka.
}

*Corresponding Author: mukunthan1989@gmail.com

\begin{abstract}
Remote medical monitoring and consultation has become indispensable in order to enhance the availability of better health-care services to the patients in remote rural areas in the country. This paper proposes an inexpensive, easy to handle Remote Medical Consultation System (RMCS) which supports the healthcare workers to carry out their services through bi-directional video and voice communication between the remote end and doctor's end as well as automated measuring of medical parameters that can be controlled from both ends. RMCS is consisted of a wearable sensors kit, a centralized hardware platform which connects to the medical sensors and devices and a software platform with database for operating and managing the system. RMCS is capable of remotely measuring patient's blood pressure, heart rate, body temperature, electrocardiogram (ECG), heart sounds and the system's platform supports to add-on more medical sensors or devices. The key aspect of the system is that it reduces most of the complexity in operation and facilitates the doctors to monitor and diagnose the patients in real-time. RMCS was essentially developed to eliminate the issues of low quality healthcare services in rural areas and to assist in monitoring immobilized patients.
\end{abstract}

Keywords: healthcare; remote medical care; telemedicine; tele-monitoring; body-sensor network (BSN)

\section{INTRODUCTION}

Telemedicine is currently being used world-wide to bridge the physical distance and time between patients in remote areas and medical specialists around the world. One of the early examples of telemedicine application was at Massachusetts General Hospital (MGH) and Boston's Logan airport in 1968 to eliminate the need of permanent doctor assignment to airport [1]. In Sri Lankan context, World Health Organization (WHO) initiated a project in 2003 called Health Telematics (SRLHT) with the purpose of obtaining advanced medical consultation regarding a patient's symptoms from other doctors in any of the eight hospitals in five districts where the telemedicine was implemented [2]. SRLHT provided the fundamental facility of sharing information about patients and their symptoms and it was augmented with Voice over Internet Protocol (VoIP) for real time communication during tele-consultation sessions.

The developing countries like Sri Lanka and other South Asian countries can greatly utilize their well-established telecommunication infrastructure to implement low-cost, sustainable telemedicine solutions for the local delivery of primary health care. As Sri Lanka still has higher number of people in rural areas [3], lack of medical resources such as only 3.8 hospital beds per 100,000 population [4] and various adverse factors affecting patients from rural areas such as high transportation cost and physical fatigue caused by 
long journeys, such telemedicine systems could greatly enhance the quality of health care services provided to the rural people of the country. This paper presents a low cost, light weight and less power consumable Telemedicine Kit which consists of a wearable sensors jacket, power supplies, high durable case and communication modules that serves as a robust portable mobile system which is suitable for developing countries. The system has the capability of remotely measuring and recording key medical parameters such as body temperature, heart sounds, pulse rate, blood pressure and ECG. A desktop software application at remote patient's end and a web-based application at the doctor's end provide the basic remote monitoring and measuring capability for the system. Also the system is capable of transmitting bi-directional video feed and uni-directional heart sounds audio feed that makes the kit an effective initial diagnostic tool. Special feature of this kit other than general telemedicine kit is that the doctor's ability to control remote end devices from doctor's end and its use as a medical parameter storage tool which can be used to access past medical records and treatments of a patient for diagnostic purpose.

\section{OUTLINE}

The Telemedicine Kit is consisted of a portable computer and several medical peripherals: a digital stethoscope, sphygmomanometer and sensors to record body temperature and ECG. The kit allows a health care practitioner in a remote area to capture the patient's vital signs in the forms of data, audio, and images and transfer them over the internet to a doctor/specialist for diagnosis and prescriptions. A customized web based control panel developed specifically for this Telemedicine Kit allows the doctors to fully control all the functions of the kit through the web interface. Also it provides videoconferencing facility between the patient and the doctor. The system can be functionally analyzed as it is consisted of biological sensors unit, data acquisition unit, communication unit and power unit. Figure1 shows the RMCS being used at remote end.

\section{A. Hardware Platform}

The hardware platform integrates the medical sensors, devices and the data acquisition modules. It acts as an interface between the medical peripherals and the computer at remote end. For the fundamental scope of the system, the system has been equipped with a digital stethoscope, temperature sensor, ECG electrodes and digital sphygmomanometer. The centralized platform could be used to add-on more medical devices or sensors and it is stacked with an Arduino AT mega 2560 board for the connectivity with the computer.

For a healthy human being, frequency range of the heart sound is between $15-200 \mathrm{~Hz}$ and for pulmonary sounds, high pitched murmurs, clicks and ejection sounds; it is between $100-500 \mathrm{~Hz}$. Also during abnormal conditions, lung sound frequency can go nearly up to $1000 \mathrm{~Hz}$. Thus, RMCS uses the CK E600 Spirit model digital stethoscope which has multiple frequency filter modes (i.e. Bell [15 - $200 \mathrm{~Hz}$, Diaphragm [100-500Hz] and Extended [15-4000Hz]) [5] that can be utilized to record and diagnose the heart sounds and lung sounds of the patients. RMCS facilitates the doctor to request the health care worker at the remote end through video-conferencing to select filtering modes and keep the stethoscope at various points of the body in order to listen to the heart sounds in realtime through the web-based terminal at doctor's end.

Also the wearable biomedical sensors unit is composed of non-invasive blood pressure meter, temperature sensor and ECG electrodes. The computer at the remote end acquires 
the data from the wearable biomedical sensors unit through the Arduino and hardware platform [6]. The hardware platform is connected to the computer via USB interface and all the measurements could be initiated and recorded through the software interfaces on both ends.

In order to obtain a diagnostic ECG from the patient, the telemedicine kit is equipped with a 3 lead ECG unit. ECG electrodes are connected in Limb lead II method, as it is the most common monitoring lead configuration and thus helps the RMCS to get the time interval between largest positive R waves which is used to compute the ECG heart rate. ECG data is acquired from the ECG electrodes via the hardware platform and the data is preprocessed to reduce the effect from baseline wander, power line noise and high frequency noises from ECG signals. As the possible base wander noise of ECG is less than $0.05 \mathrm{~Hz}$ and the required higher frequency to see important peaks of ECG is $100 \mathrm{HZ}$, a $0.05-100 \mathrm{~Hz}$ band pass filter was built into the Arduino code. Also a $50 \mathrm{~Hz}$ notch filter was included to remove baseline wander and power line interface. In addition, ECG was sampled at rate of $200 \mathrm{HZ}$ through Arduino code to satisfy minimum requirement of Nyquist theorem [7]. As the temperature sensor connects in Wheatstone bridge in the platform, RMCS is equipped with a library that was written to calculate the actual body temperature after the calibration test had been done. In order to automate the non-invasive blood pressure measurement, a transistor switch circuit is implemented in the hardware platform by using the Arduino pin as one input to control the device. It enables the doctor to remotely control the sphygmomanometer.

\section{B. Software Platform}

\section{1) Networking:}

Communication between RMCS device and doctor's terminal is established using Internet. RMCS at remote end is capable of connecting to cloud through a mobile broadband or wireless/wired internet connection. At remote end sensors are connected to eHealth platform and Arduino which communicates with the laptop using serial communication. Arduino is interfaced using a Java desktop application which gathers the sensor outputs and upload the data to the server. At the doctor's end terminal, doctor can access the data in the server using a web based application. Video conferencing also can be established between telemedicine kit and doctor's end using Skype.

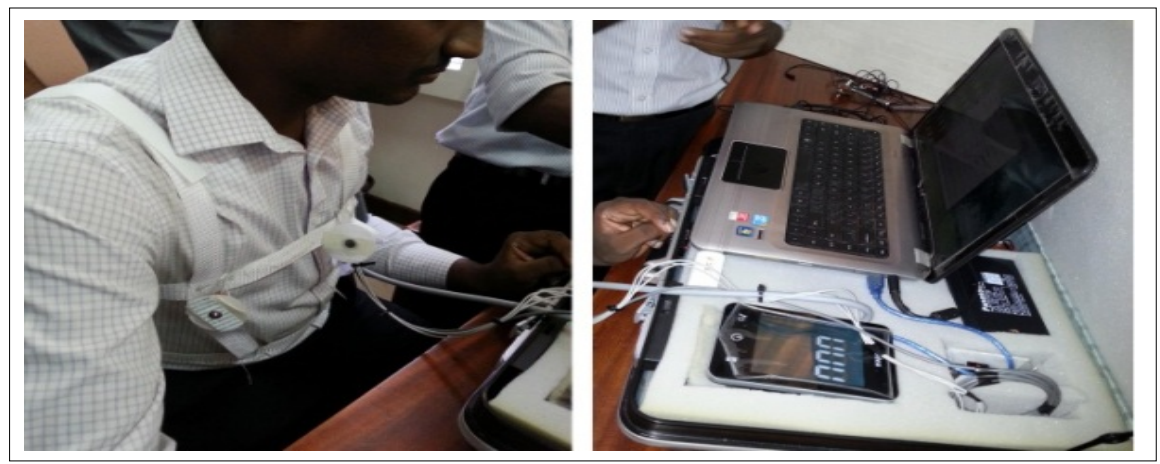

Fig. 1. Wearable sensors kit and remote-end device. 


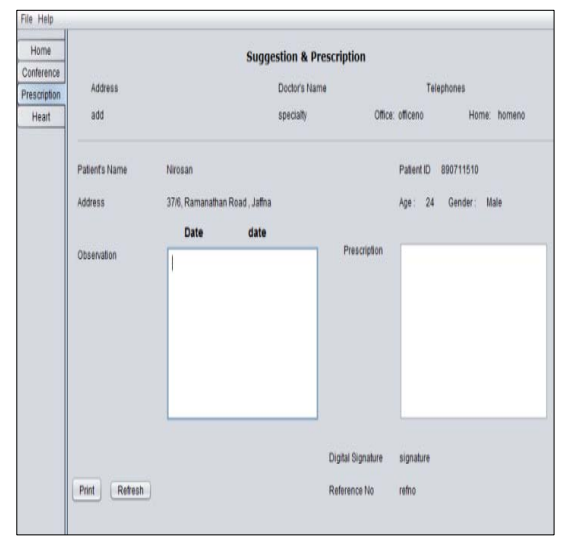

Fig. 2. Snapshot of remote end GUI showing the prescription tab

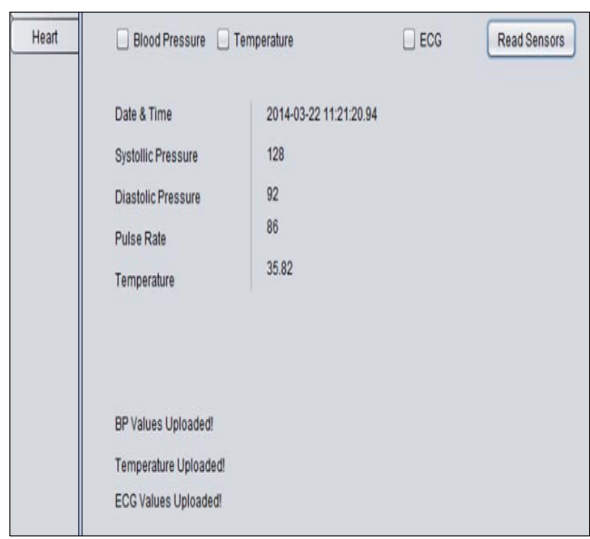

Fig. 3. Snapshot of remote end GUI showing the medical readings

In addition to that, RMCS provides an ultimate facility to the doctors by letting them control which medical parameters should be recorded and sent to the doctor's end. Also the doctor could listen to the recorded heart sounds as well as the real-time heart sound streaming from patient's end. These features save both the patients' and doctors' time and reduces the need for a third party assistant at remote end. The controlling of remote end application is carried out using database communication, where the doctor's end would update the requirement control word to a table in the database as well as listening to it continuously for the response from remote-end. The remote-end application would also listen to the database continuously over the same time and once it obtains the control word for measuring medical parameters, it runs the functions at remote end and updates the database immediately with the measured medical data. A unique synchronization id has been used in controlling table as well as in measurements tables in the database, such that the doctor's end can fetch the specific data for a particular patient on real-time.

\section{2) Remote end application:}

The remote end application was built in Java and utilized to communicate with Arduino board via serial communication and to communicate with doctor's end via internet. It was programmed to aid the health care workers at remote areas in creating, viewing, capturing and sending patient details and medical entries to the main database, sending real time heart sounds, recording heart sounds and facilitating video conference between patient's end and doctor's end. Also as the Fig. 2 shows it enables the system to immediately display the observation and prescription of the doctor on the remote end in real-time. If the doctor is available on the other end, he can take control of the telemedicine kit so as to measure the required medical parameters needed for the diagnosis of the patient. Even if the doctor is unavailable on the other end, the health care worker can still operate the telemedicine kit manually using the application. He/she can record the required medical data from the patient and it will be uploaded to the main server so that the doctor can access it and provide his suggestions later. 
Measurements from biomedical sensor unit gathered by the data acquisition unit are processed by the Arduino board and displayed in the Graphical User Interface (GUI) of the remote end system. GUI displays the measurements in an organized manner and carries out the data communication over the internet. In addition, there will be an option to perform video conferencing between patient's end and doctor's end. Figure 3 shows the sample readings in the GUI at the patient's end. In the current system, body temperature, blood pressure, pulse rate and ECG parameters are uploaded to database which can be accessed by the doctor immediately. Furthermore, near real time audio streaming is achieved using a client server program with VLC Media library given by Video LAN cooperation. Also the server is capable of storing the audio streams in the database [8].

At the patient's end, streaming client capture the audio from electronic stethoscope, transcode it as 320kbps CBR mp3 with 8000 samples per second. The Streaming server establishes a connection with the client when communication is initiated. Advanced Stream Redirector (ASX) in the server redirects the incoming stream to an output port. Once the redirection is done, stream will be available at the doctor's end. Using the above technology, near real time audio streaming with 2-3 second network latency was achieved.

Thus, once the doctor has accessed and observed all the medical data from the patient, he can provide his suggestions and prescriptions in tandem. Doctor's prescription will appear on that GUI itself so that patient can print it out. These readings and responses are sent via the internet to a server. The database structure at the server facilitates the doctors to access a patient's medical history as well as the latest medical readings in order to make the medical diagnosis.

3) Doctor's end application:

The doctor's end application was built using PHP and AJAX technologies, Such that it offers cross platform interoperability by letting any internet enabled web browsers to access the services provided by the RMCS web server. It was built on top of a light weight open source PHP framework called Code Igniter to provide secure database access as well as quickly loading swift system. As the Fig. 4 shows the web based application provides a comprehensive control panel that enables the doctor to control the procedure of taking real time measurements using the sensors remotely. In the same panel, the doctor can choose the medical parameters to be measured, access patient's medical history and listen to pre-recorded heart sounds as well as real-time streaming of the heart sounds from remote end. Also as depicted in Fig. 5 the interface assists the doctor to see patient's previous medical history as graphical representation and to suggest the prescription hassle-free. The doctor's end application could be accessed via any web browsers as well as smart phone mobile browsers which additionally provides mobility on both doctor's and patient's ends.

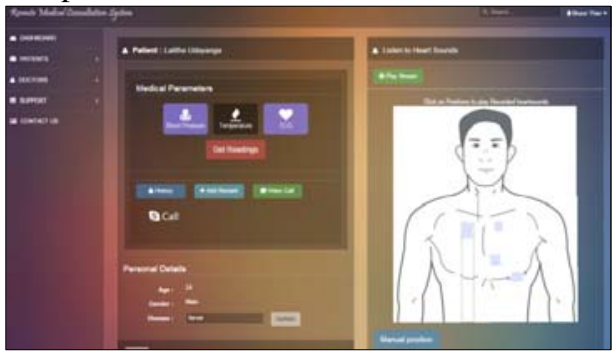

Fig. 4. Snapshot of doctor's end Control Panel GUI

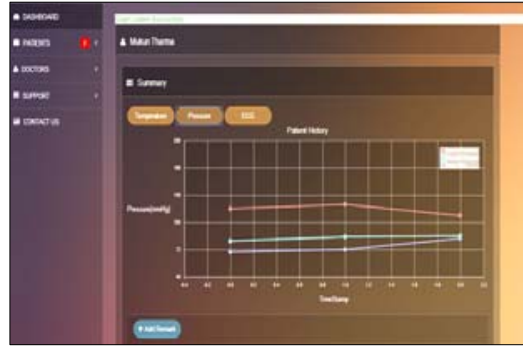

Fig. 5. Snapshot of doctor's end GUI: Patients 
RMCS was mainly implemented to be mostly used in remote and rural areas where the possibility of adequate power sources could be questionable. Therefore, the power unit of the RMCS is consisted of a customized AC adapter that is used with a $230 \mathrm{~V}$ main supply and a $12 \mathrm{~V}$ rechargeable battery is integrated into the power unit as a backup in the absence of utility power. This ensures the portability and mobility of the kit. The customized AC adapter converts the $230 \mathrm{~V}$ to $12 \mathrm{~V}$ for the operation of the Arduino board and the sensors. Also the Arduino prevents over-charging of the battery by controlling the charging circuit based on the output voltage of the battery.

\section{DisCUSSIONS AND CONCLUSION}

The current implementation of the project is consisted of the prototype of the TeleMedicine kit with controller applications for remote end and doctor's end. The kit could measure the medical parameters such as body temperature, blood pressure, ECG and pulse rate where the data is stored in a relational database system. Patient's history can be analyzed at the doctor's end for further diagnosis. The ability of letting the doctor control the telemedicine kit adds up value to the RMCS as it reduces the unnecessary time taken to record all the medical parameters of the patients without the real requirement for it. Also the two way audio-video communication between the doctor's end and patient's end and the immediate medical data transfer and doctor's response helps the system to be a real time solution and serves the purpose of the system. Achieving a nearly real-time streaming of heart sounds provides more information for the doctors to narrow down their diagnosis and provide better quality of service to the patients. Also in case of low network coverage in rural areas, the system can exploit the normal heart sound recording functions at remote end, such that the doctor can still listen to the heart sounds for diagnosis.

On the other hand, the current system uses a laptop for the data processing and video conferencing facilities which adds a drawback to the mobility and cost of system. But it can be replaced with a mini tablet pc with the software modified as a mobile application. In terms of adding on more medical devices or sensors, most of the proprietary standard medical sensors do not allow for interfacing to external equipment, such that it limits the extendibility of the system. Another constraint is that the health care workers should have sufficient knowledge in handling the sphygmomanometer and the stethoscope in order to get proper medical readings without failures.

\section{FUTURE WORKS}

The proposed kit measures basic medical parameters such as blood pressure, body temperature, ECG and pulse rate. This system could be extended by measuring more important medical parameters using devices such as glucometer, pulse oximeter, Electromyography etc. Also the existing one to one communication between remote patient's end and doctor's end can be extended to support one to many or many to many communication protocols. Using Raspberry pi cluster or android platforms could make the system less weighted, less power consuming and more portable. Addition of more features such as automated heart sound anomaly detection, automated alerts during critical conditions can be achieved by integrating an expert system into the web-based interface. Also this tele-medicine system could be implemented along with telepresence robots to carry out health care services in rural areas, disaster situation or even as a paramedical kit. 


\section{Acknowledgment}

The authors would like to thank Arthur C Clarke Institute for Modern Technologies (ACCIMT) for providing the initial medical equipment and continuous support to carry out the project successfully.

\section{References}

[1] Ari T. Adler (2000), “A cost-effective portable telemedicine kit for use in developing countries, Massachusetts Institute of Technology”, [Online]. Available:

http://resenv.media.mit.edu/ pubs/theses/AriAdler-Thesis.pdf [Accessed: October 27, 2013].

[2] Dr. Palitha Gunawardhana (2003, November). "Telemedicine implementation experience in Sri Lanka”. Center for Telemedicine General Hospital Kandy, Sri Lanka [Online]. Available: http://www.unoosa.org/pdf/sap/2006/india2/ presentations/5_3.pdf) [Accessed: November 20, 2013].

[3] Trading Economics, Rural population in Sri Lanka [Online]. Available: http://www.tradingeconomics.com/sri-lanka/rural-population-wb-data.html . [Accessed: October 27, 2013].

[4] Medical statistics unit (2012). Annual Health Bulletin, Ministry of Health, Sri Lanka [Online]. Available: http://www.health.gov.lk/en/publication/AHB-2012/Annual \%20Health\%20Bulletin\%20-\%202012.pdf. [Accessed: October 27, 2013].

[5] Spirit E600 Precision Electronic Stethoscope[Online], Available: http://www.medscope.co.uk/Spirit_E600_Precision_Electronic_Stethoscope pp 9498.htm [Accessed: October 27, 2013]

[6] Cooking Hacks [Online]. Available: http://www.cookinghacks.com/documentation/tutorials/ehealth-biometric-sensor-platform-arduino-raspberry-pimedical [Accessed: October 29, 2013].

[7] Tompkins, W.J., Biomedical Digital Signal Processing, [Online]. Available: https://ay1415.moodle.wisc.edu/prod/pluginfile.php/72917/mod_resource/ content/1/BME\%20DSP\%20textbook.pdf [Accessed: March 15, 2014]

[8] Streaming Documentation, VideoLAN Organization, [Online] Available: http://www.videolan.org/vlc/streaming.html [Accessed: March 15, 2014] 